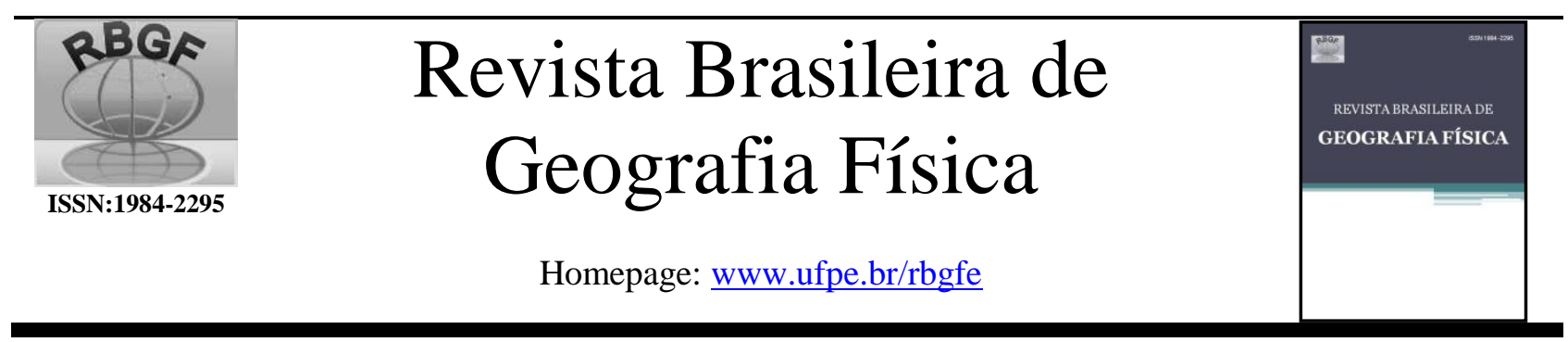

\title{
Análise de sensibilidade e calibração espacial do modelo SWAT aplicado em uma bacia do litoral pernambucano através de dados climáticos observados e de reanálise
}

\author{
Yenê Medeiros Paz ${ }^{1}$, Josiclêda Domiciano Galvíncio² ${ }^{2}$ Romildo Morant de Holanda ${ }^{3}$, Raghavan Srinivasan ${ }^{4}$, \\ Charles Allan Jones ${ }^{5}$
}

\begin{abstract}
${ }^{1}$ Doutoranda pelo Programa de Desenvolvimento e Meio Ambiente (Prodema) da Universidade Federal de Pernambuco (UFPE), Av. Prof. Moraes Rego, 1235, Cidade Universitária, CEP 50670-901, Recife, Pernambuco. yenemedeiros@ hotmail.com (autor correspondente). ${ }^{2}$ Professora Dra., Departamento de Ciências Geográficas da UFPE, josicleda@ gmail.com. ${ }^{3}$ Professor Dr., Departamento de Tecnologia Rural, Universidade Federal Rural de Pernambuco (UFRPE), Rua Manuel de Medeiros, s/n, Dois Irmãos, CEP 52171-900, Recife, Pernambuco. ${ }^{4}$ Professor in the Departments of Ecosystem Sciences and Management and Biological and Agricultural Engineering, Texas A\&M University, College Station, Texas. ${ }^{5}$ Senior Research Scientist, Texas A\&M University, College Station, Texas.
\end{abstract}

Artigo recebido em 09/11/2017 e aceito em 24/02/2018

\section{R E S U M O}

O modelo hidrológico SWAT está sendo bastante difundido no Brasil e devido necessidade de ajustes nos resultados obtidos através de modelagens e dados observados, implica-se na realização de uma calibração. Assim, o objetivo do presente trabalho foi realizar a calibração espacial de um modelo SWAT para a bacia do rio Goiana em Pernambuco, utilizando-se dados climáticos observados e de reanálise. Foram utilizadas nove estações pluviométricas, cujo percentual de falhas para o período utilizado na simulação variou de 0 a $14.19 \%$, e nove estações de dados de reanálise obtidos através do CFSR. A análise de sensibilidade e calibração foi realizada em cinco sub-bacias. Verificou-se que o uso de dados observados de estações locais garantiu um maior número de estações satisfatoriamente calibradas. Contudo, fazendo-se uso de dados climáticos locais mais globais houve uma melhora das estatísticas de sub-bacias cujas estações pluviométricas estavam melhores distribuídas. As estatísticas alcançadas na modelagem desenvolvida no SWAT evidenciam a aplicabilidade do modelo para estimativas mensais de vazão. Este demostrou boas predições para o exutório principal, contudo restrições para a área mais seca da bacia, cujos resultados foram insatisfatórios.

Palavras-chave: vazão, modelagem hidrológica, SWAT, dados climáticos.

\section{Sensitivity analysis and calibration of the SWAT model for a basin in northeastern Brazil using observed and reanalysis climatic data}

\section{A B S T R A C T}

The hydrological model SWAT is very widely used in Brazil, and calibration is needed to adjust results obtained through modeling to observed data. This article describes the spatial calibration of a SWAT model for the Goiana river basin in Pernambuco, using observed and reanalyzed climatic data. Nine rainfall stations were used, with the percentage of gaps in the simulation ranging from 0 to $14.19 \%$. Nine stations of reanalysis climatic data obtained through the CFSR were used. The sensitivity and calibration analyses were performed in five subbasins. Use of observed data from local stations produced a greater number of satisfactorily calibrated stations. However, using both local stations and reanalysis climate data produced better statistics for subbasins with few rainfall stations that also had substantial missing data. The statistics obtained demonstrated the applicability of the model to monthly flow estimates. Even though model predictions for the driest area of the basin were unsatisfactory, the calibrated model provided good predictions for the main basin outlet. Keywords: streamflow, hydrological modeling, SWAT, climatic data. 


\section{Introdução}

Como descrevem Medeiros e Silva (2014) os modelos matemáticos estão em ascensão e têm grande importância em estudos climáticos, tendo os custos de determinação dos resultados inferiores. Apesar das incertezas e dos processos probabilísticos, os modelos hidrológicos se constituem de uma metodologia capaz de avaliar os processos a nível de bacia hidrográfica, realizandose projeções de condições futuras (Praskievicz e Chang, 2009). Esses são utilizados para previsão de cheias e inundações, gestão dos recursos hídricos, avaliação da qualidade de água, erosão, sedimentação, uso e ocupação do solo, mudanças climáticas, circulação de nutrientes e pesticidas, entre outros (Devi, Ganasri e Dwarakish, 2015). Para Engel et al. (2007) um modelo se refere a uma ferramenta que pode ser usada para analisar uma determinada hipótese, não sendo a própria hipótese em si. Assim, sua utilização é fundamental para testar e avaliar mudanças a partir de análises espaço-temporais devido a distribuição espacial e temporal dos fenômenos hidrológicos (Pontes et al., 2016; Blainsk, Acosta e Nogueira, 2017).

Diversos modelos têm sido desenvolvidos ao longo do tempo e discutidos em diversos trabalhos, como o SWAT Model (Soil and Water Assessment Tool), MIKE SHE model (Systeme Hydrologique European), HBV model (Hydrologiska Byrans Vattenavdelning model), TOPMODEL, VIC model (Variable Infiltration Capacity model), BASINS (Better Assessment Science Integrating point \& Non-point Sources) (Praskievicz e Chang, 2009; Devi, Ganasri e Dwarakish, 2015). Um modelo que está sendo bastante difundido no Brasil é o SWAT (Arnold et al., 1998) que pode ser caracterizado como um modelo hidrológico semi-distribuído que realiza operações diárias, capaz de predizer vazão, escoamento superficial, produção de sedimentos, qualidade das águas a partir de modificações no uso e ocupação do solo de bacias hidrográficas (Gassman et al., 2007; Arnold et al., 2012a).

De acordo com Veith et al. (2010) visto que as simulações dos modelos hidrológicos apresentam resultados com variação em relação aos dados observados, verifica-se a necessidade da realização da calibração desses modelos. Diversos trabalhos foram desenvolvidos apresentando a aplicabilidade do modelo SWAT, que com o uso da calibração manual ou automática pode alcançar modelagens bastante satisfatórias como mostram alguns trabalhos recentes (Lelis et al., 2012; Pereira et al., 2014a; 2016; Santos et al., 2014; 2015; Bressiani et al., 2015; Fukunaga et al., 2015).
A análise de sensibilidade vem sendo realizada em diversos trabalhos como etapa anterior a calibração, visando encontrar os parâmetros que forneçam as melhores respostas (Feyereisen et al., 2007; Lelis et al., 2012). Esses parâmetros são ajustados tendo-se uma compreensão das características da bacia hidrográfica e dos processos envolvidos, analisando-se valores reais de medições em campo e outras fontes de informação (Daggupati et al., 2015a; Brighenti, Bonumá e Chaffe, 2016). Para Sarrazin et al. (2016) a análise de sensibilidade caracteriza o impacto da modificação dos dados de entrada nos dados de saída. Segundo Engel et al. (2007) e Nossent e Bauwens (2012) esta análise permite verificar a influência de certos parâmetros, identificando-se quais inputs são mais importantes na modelagem. Nossent et al. (2011) ainda destaca a importância da análise na verificação de efeitos de interação entre os parâmetros.

$\mathrm{Na}$ maioria dos trabalhos brasileiros verifica-se que a estratégia de análise de sensibilidade, calibração e validação utilizada faz uso de apenas um outlet, como verifica-se nos trabalhos de Andrade, Mello e Beskow (2013), Pereira et al. (2014a; 2014b), Rodrigues et al. (2015), Brighenti, Bonuma e Chaffe (2016), e Franco e Bonumá (2017), contudo verificam-se pesquisas que trabalham de maneira especializada como o de Lelis et al. (2012), Bressiani et al. (2015) e Eduardo et al. (2016). A calibração através de outlet, como indica Daggupati et al. (2015a) pode ser bem aplicada para bacias pequenas com características uniformes, mas para bacias maiores os resultados resultarão em valores médios da bacia que podem ser superestimados ou subestimados em vários pontos, sendo indicada a calibração espacial. Os resultados da calibração ainda devem ser analisados com cautela pois como aponta Abbaspour (2005) existem muitas soluções durante o processo de calibração e o usuário deve analisar qual se adequa as condições da área de estudo.

No contexto de dados climáticos observados brasileiros, verifica-se a presença de falhas, dessa maneira, buscam-se alternativas para estudar os processos hidrológicos. Problemáticas referentes aos inputs foram registradas no trabalho de Dile e Srinivasan (2014) e Bressiani et al. (2015) onde os autores utilizaram dados climáticos globais de reanálise a fim de comparar com a eficiência no uso de dados observados locais que apresentaram falhas. Apresentando-se como uma opção para bacias hidrográficas cujas estações estejam mal 
distribuídas ou com problemas relativos à informações.

Dessa maneira, o objetivo do presente trabalho foi realizar a calibração espacial de um modelo SWAT para a bacia do rio Goiana em Pernambuco, utilizando-se dados climáticos observados e de reanálise.

\section{Material e métodos}

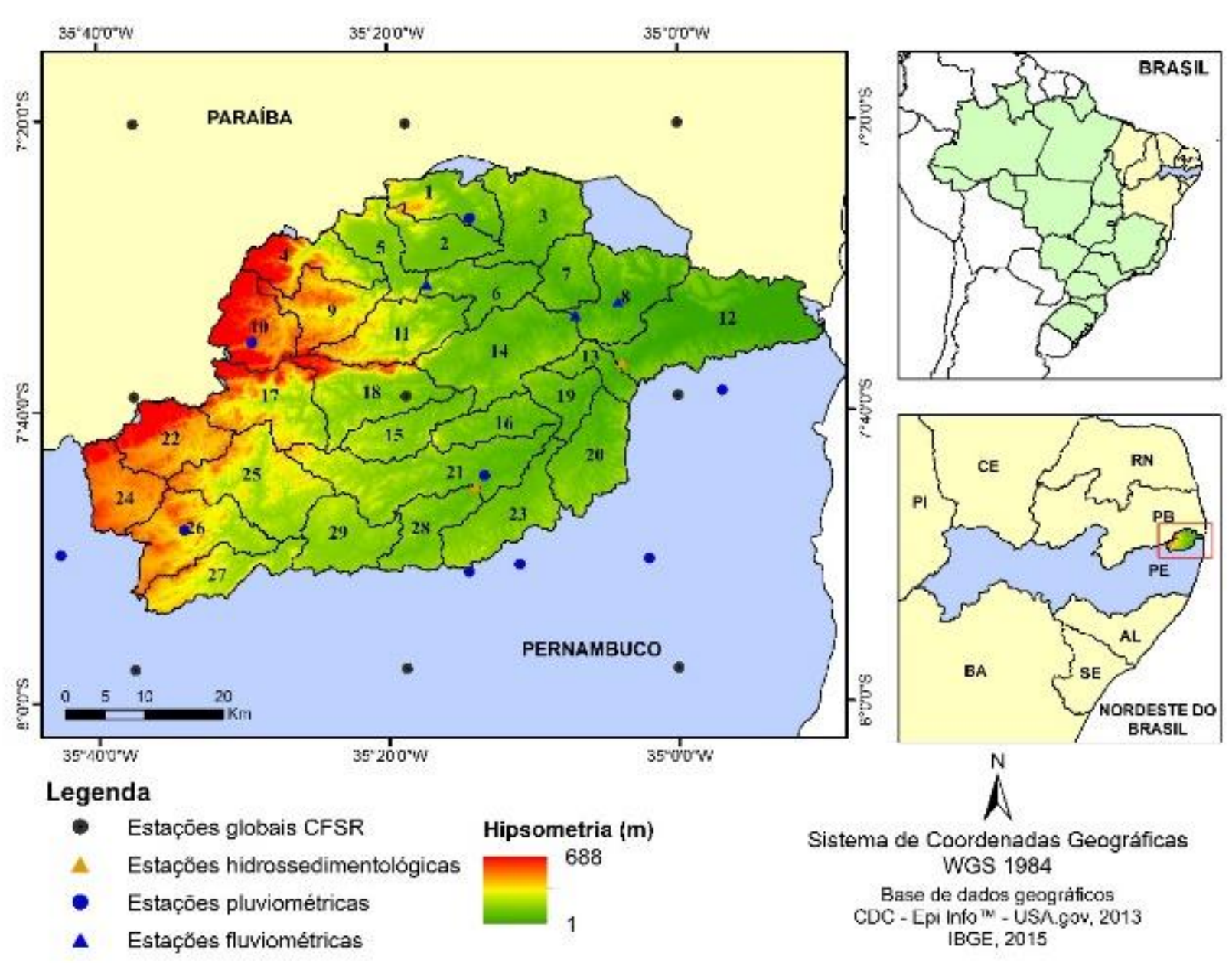

\section{Caracterização da área de estudo}

A bacia do rio Goiana possui uma área de 2.847,53 $\mathrm{km}^{2}$, localizada entre a latitude $07^{\circ} 22^{\prime} 20^{\prime}$ ' e $07^{\circ} 54^{\prime} 47^{\prime}$ ' sul e longitude entre $34^{\circ}$ 49'06', e 35'41'43', oeste, contemplando 26 municípios da zona da mata norte, agreste setentrional e metropolitana (Figura 1).

Figura 1. Localização da bacia do rio Goiana no nordeste brasileiro e estações hidroclimáticas utilizadas.

É composta pelas sub-bacias do rio Capibaribe Mirim, Tracunhaém e a do Goiana stricto sensu. A bacia do rio Goiana possui limites ao norte com o estado da Paraíba e o grupo de bacias de pequenos rios litorâneos 6 , na parte sul possui limites com a bacia do rio Capibaribe e o grupo de bacias de pequenos rios litorâneos 1 , na parte leste com o Oceano Atlântico e a oeste também com o estado da Paraíba (APAC, 2016). A elevação da bacia inicia-se no nível do mar e atinge $688 \mathrm{~m}$ em seu maior pico nas proximidades com o estado da Paraíba. Fora do Litoral Norte, a maior parte da bacia é localizada em terrenos do Embasamento Cristalino, dependendo da pluviosidade para o abastecimento de sua rede hidrográfica (CPRH, 2003).

Como principais usos da água na região, tem-se a exploração da carcinicultura e pesca, consumo humano, abastecimento público $\mathrm{e}$ industrial, consumo animal, turismo, recreação e lazer. $\mathrm{E}$ os principais fatores impactantes aos recursos hídricos podem ser pontuados como descarga de efluentes domésticos, industriais e agroindustriais, retirada de areia do leito dos rios, construção de edificações nas proximidades dos cursos de água, captação de água do leito dos rios não regulamentada, desmatamento das matas ciliares e nascentes dos rios e lançamento de lixo 
diretamente no rio por parte da população (CONDEPE/FIDEM, 2005).

\section{Modelo SWAT}

O modelo foi desenvolvido pela USDA Agricultural Research e Texas A\&M University e possui diversos componentes como hidrologia, informações climáticas, sedimentação, caracterização dos solos, crescimento de culturas, detalhamento de nutrientes e pesticidas, assim como manejo de culturas agrícolas (Arnold et al., 2010). O modelo SWAT ainda possui duas divisões nas simulações hidrológicas, consistindose a primeira fase no ciclo hidrológico e a segunda como a fase do encaminhamento da água (Sarrazin et al., 2016)

O nível de discretização adotado pelo modelo se refere as Unidades de Resposta Hidrológica (Hydrologic Response Units - HRU) como apontam Arnold et al. (2010). Estas se referem a áreas homogêneas em relação ao tipo de solo, uso e cobertura, topografia e manejo e não são identificadas espacialmente (Gassman et al., 2007). As diferentes variáveis que o modelo é capaz de estimar, assim como a caracterização dos parâmetros e equações utilizadas para o funcionamento do modelo são descritas na documentação teórica disponibilizada online (Neitsch et al., 2011). O modelo é baseado na equação do balanço hídrico:

$$
\mathrm{SW}_{\mathrm{t}}=\mathrm{SW}_{0}+\sum_{i=1}^{t}\left(\mathrm{R}_{\mathrm{day}}-\mathrm{Q}_{\text {surf }}-\mathrm{E}_{\mathrm{a}}-\mathrm{W}_{\text {seep }}-\mathrm{Q}_{\mathrm{gw}}\right)
$$

Onde $\mathrm{SW}_{\mathrm{t}}$ se refere ao conteúdo final de água no solo $\left(\mathrm{mm} \mathrm{H}_{2} \mathrm{O}\right), \mathrm{SW}_{0}$ é o conteúdo inicial de água no solo no dia $\mathrm{i}\left(\mathrm{mm} \mathrm{H}_{2} \mathrm{O}\right)$, té o tempo em dias, $\mathrm{R}_{\text {day }} \mathrm{o}$ quantitativo de precipitação no dia i $\left(\mathrm{mm} \mathrm{H}_{2} \mathrm{O}\right)$, Q $\mathrm{Q}_{\text {surf }}$ é a quantidade de escoamento superficial no dia i $\left(m m \mathrm{H}_{2} \mathrm{O}\right), \mathrm{E}_{\mathrm{a}}$ é a evapotranspiração no dia i (mm H2O), Wseep é a percolação no dia i $(\mathrm{mm})$, e Qgw é o quantitativo do fluxo de retorno no dia i $\left(\mathrm{mm} \mathrm{H}_{2} \mathrm{O}\right)$.

Dentre os métodos disponibilizados para estimativa da evapotranspiração potencial no modelo (Neitsch et al., 2011), selecionou-se Penman-Monteith, que segundo Gassman et al. (2007) deve ser usado para cenários de mudanças climáticas em relação a níveis de $\mathrm{CO}_{2}$. E para prever o escoamento superficial da chuva para diferentes tipos de solo e cobertura da terra, o modelo utiliza o método da equação da curva número (CN; Arnold et al., 1998) em função da umidade solo. Para este trabalho foi utilizado o ArcSWAT versão 2012 (revisão 658) com a interface do ArcGIS 10.2.2 (ArcSWAT).

\section{Dados de entrada e configuração do modelo}

$\mathrm{O}$ modelo SWAT requer dados de solos, uso e cobertura, modelo de elevação digital (DEM) e clima para realizar uma simulação hidrosedimentológica (Figura 2). E a resolução tem importância na predição de vazão e sedimentos, sendo o DEM o input mais sensitivo (Cotter et al., 2003).

A bacia do goiana foi delineada a partir do modelo de elevação digital do terreno e do posicionamento de outlets para delimitação das sub-bacias. Assim, obteve-se 29 sub-bacias cujas áreas variam de 36,94 a 218,07 km² com média de $98,85 \mathrm{~km}^{2}$.

O modelo de elevação digital do terreno foi obtido através do monitoramento por satélite da Embrapa (www.cnpm.embrapa.br) que disponibiliza imagens do Shuttle Radar Topographic Mission (SRTM) com resolução de $90 \mathrm{~m}$ para todo o Brasil.

O mapeamento dos solos da região foi realizado através do Zoneamento Agroecológico de Pernambuco (ZAPE) da Embrapa, com uma escala de 1:100.000. A classificação dos solos seguiu o Sistema Brasileiro de Classificação de Solos (SiBCS; EMBRAPA, 2013). O levantamento das características físicas e químicas das camadas dos solos foi realizado através do Sistema de informação de solos brasileiros (SiSolos) da Empresa Brasileira de Pesquisa Agropecuária (Embrapa) como profundidade, textura e carbono orgânico. Demais parâmetros não mensurados foram estimados a partir de equações de pedotransferência. A classificação hidrológica dos solos foi feita através do trabalho de Sartori, Lombardi Neto e Genovez (2005).

O mapa de uso e cobertura do solo foi obtido a partir do Projeto de conservação e utilização sustentável da diversidade biológica brasileira (Probio) coordenado pelo Ministério do Meio Ambiente (MMA). Este mapeamento foi elaborado numa escala de 1:250.000, tendo ano base de mapeamento o ano de 2002, utilizando-se cenas de 1999 a 2005, concentrando-se a maior parte das imagens do ano de 2001-2003 e utilizando-se cenas a partir do sensor ETM+ do Landsat 7, SPOT4 e CCD/CBERS (IESB, 2007).

A partir das classes de uso e cobertura do mapa do Probio foi realizado uma análise das classes disponibilizadas pelo SWAT visando atribuir novas categorias. Este procedimento foi realizado observando-se as características das áreas, de modo que o SWAT ainda não possui uma classificação detalhada para regiões tropicais.

A predição da vazão foi realizada com saídas mensais e a simulação foi realizada para um 
período de 10 anos, iniciando-se em janeiro de 1996 com 3 anos de aquecimento.
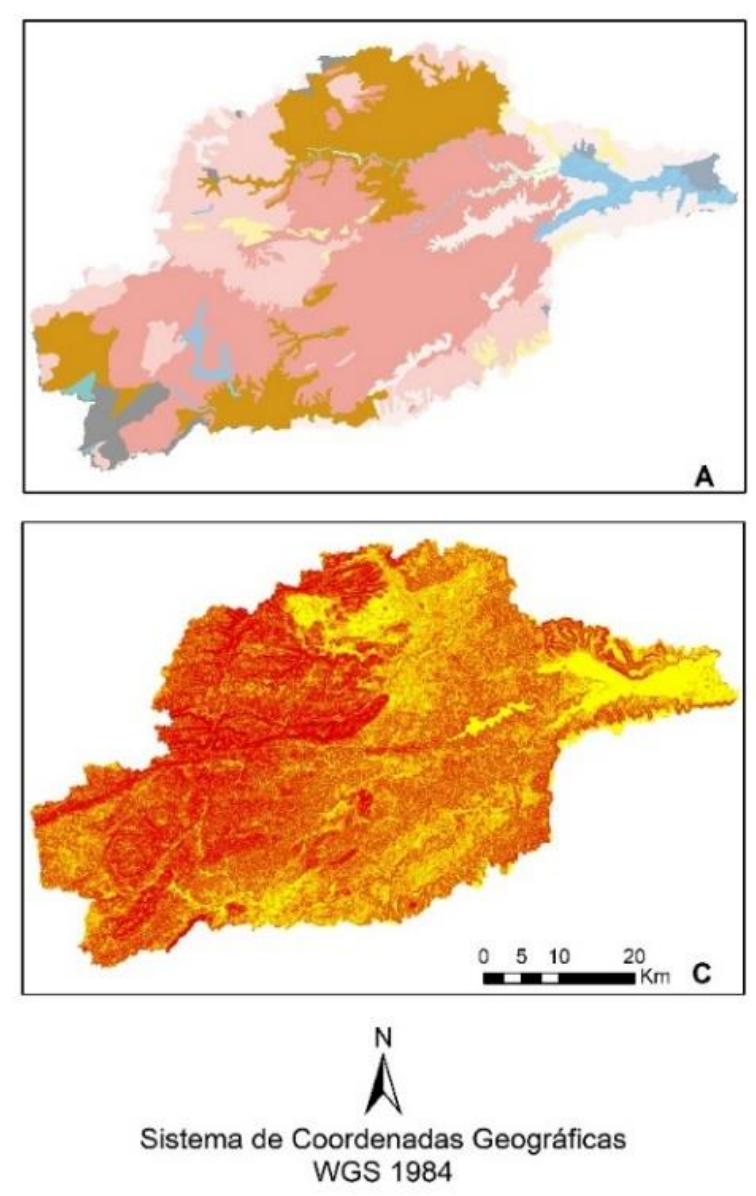

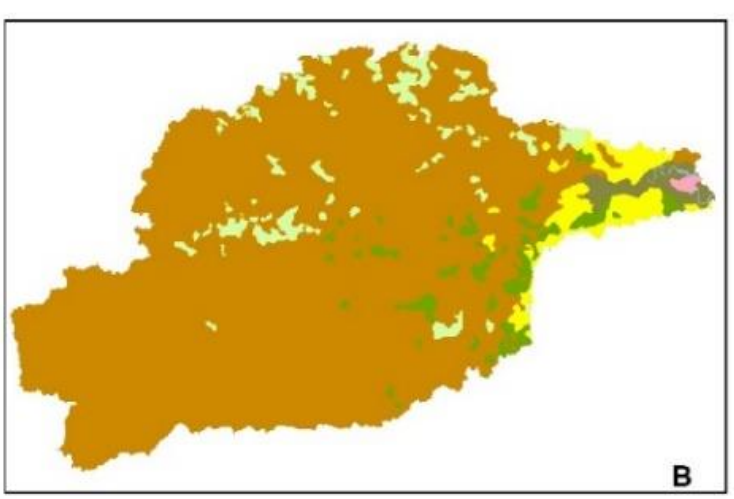

Legenda

Classes de solos
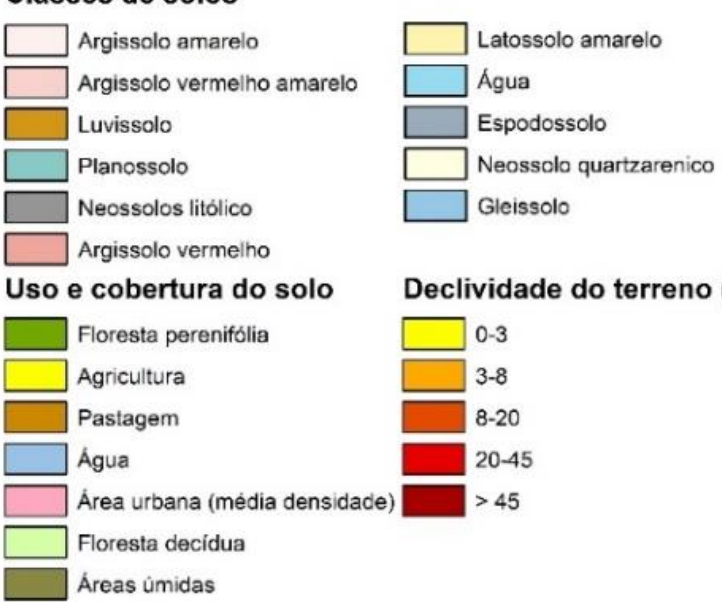

\section{Declividade do terreno (\%)}
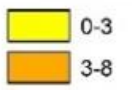

$8-20$

$20-45$

$>45$
45

Figura 2. Mapas de classes de solos (A), uso e cobertura do solo* (B) e declividade do terreno (C) da bacia do rio Goiana.

*Baseado nas classes do SWAT

\section{Dados Hidroclimáticos}

Como entrada de dados climáticos foram utilizadas 3 estações automáticas do Instituto Nacional de Meteorologia (Inmet) para o Weather Generator (WGN) (Tabela 1). Para o qual foram utilizados dados de cerca de 50 anos de precipitação, temperatura máxima e mínima do ar, umidade, radiação solar e velocidade do vento.

Tabela 1. Estações utilizadas para o Weather generator a partir de estações do Inmet

\begin{tabular}{llll}
\hline Código & Nome & Latitude & Longitude \\
\hline 82797 & Surubim & $-7,83$ & $-35,71$ \\
82900 & Recife & $-8,05$ & $-34,95$ \\
82798 & João Pessoa & $-7,1$ & $-34,86$ \\
\hline
\end{tabular}

Estações pluviométricas com dados diários disponíveis foram obtidas a partir do Inmet, Agência Nacional das Águas (ANA) e Agência Pernambucana de Águas e Clima (Apac), cujo percentual de falhas para o período utilizado na simulação variou de 0 a $14.19 \%$ (Tabela 2). A distribuição das estações não foi homogênea devido à dificuldade de selecionar estações com percentual de falhas satisfatório (Figura 1).

$\mathrm{O}$ período com menor incidência de falhas foi selecionado para a simulação (1999-2009), em virtude também da disponibilidade de dados observados de vazão para esse mesmo período. 
Revista Brasileira de Geografia Física v.11, n.1, (2018) 371-389.

Tabela 2. Estações pluviométricas utilizadas para simulação hidrológica no período de 1999 a 2009

\begin{tabular}{|c|c|c|c|c|c|c|}
\hline Código & Nome & Latitude & Longitude & Altitude & $\begin{array}{l}\text { Percentual } \\
\text { de falhas } \\
1999 \quad a \\
2009(\%)\end{array}$ & $\begin{array}{l}\text { Média anual de } \\
\text { precipitação } 1999 \text { a } \\
2009(\mathbf{m m})\end{array}$ \\
\hline 28 & Goiana (Itapirema) & $-7,6442$ & $-34,9489$ & 87 & 6,82 & 2045,75 \\
\hline 95 & $\begin{array}{l}\text { Carpina (est. exp. de } \\
\text { Cana-de-açúcar) }\end{array}$ & $-7,8511$ & $-35,2408$ & 183 & 0,15 & 1300,37 \\
\hline 97 & Nazaré da mata & $-7,7408$ & $-35,2228$ & 82 & 7,07 & 1008,58 \\
\hline 139 & Bom jardim & $-7,8017$ & $-35,5678$ & 340 & 0,32 & 784,82 \\
\hline 269 & Igarassu (Bar.catuca) & $-7,8364$ & $-35,0336$ & 50 & 4,80 & 1347,98 \\
\hline 271 & São Vicente Ferrer & $-7,5875$ & $-35,4889$ & 427 & 0,37 & 1084,79 \\
\hline 457 & Ferreiros & $-7,4461$ & $-35,2386$ & 93 & 14,19 & 676,42 \\
\hline 82797 & Surubim & $-7,83$ & $-35,71$ & 418 & 0,17 & 621,66 \\
\hline 735157 & Carpina & $-7,8428$ & $-35,1825$ & 102 & 0,00 & 1456,24 \\
\hline
\end{tabular}

\section{Dados de reanálise}

Sabendo que a distribuição e disponibilidade de dados é muito importante para a modelagem hidrológica (El-Sadek et al., 2011) e verificando que os dados disponíveis de precipitação na bacia do rio Goiana não se encontram satisfatoriamente distribuídos, foram utilizados dados climáticos globais de reanálise. Estes foram obtidos através do Global Weather Data for SWAT (http://globalweather.tamu.edu/) disponibilizado pela Texas A\&M University a fim de comparar os resultados com as estações locais descritas acima, visto que alguns trabalhos demonstraram anteriormente a aplicabilidade desses dados (Dile e Srinivasan, 2014; Fuka et al., 2014; Bressiani et al., 2015).

Os dados CFSR disponibilizados são diários e sem falhas para o período de 1979 a 2014, possuindo uma resolução de $38 \mathrm{~km}\left(0.3125^{\circ}\right)$ e cobertura global. Tendo informações de precipitação, temperatura máxima e mínima, umidade, radiação solar e velocidade do vento que são adequadas para o período simulado com os dados convencionais. Foram utilizadas 9 estações que correspondem a um box de latitude $-8,4669$ a 7,0464 e longitude $-35,777$ a $-34,585$ (Tabela 3).

Tabela 3. Estações climáticas CFSR para simulação hidrológica no período de 1999 a 2009

\begin{tabular}{llll}
\hline Estação & Latitude & Longitude & $\begin{array}{l}\text { Média anual de } \\
\text { precipitação 1999 a } \\
\mathbf{2 0 0 9}(\mathbf{m m})\end{array}$ \\
\hline p-80-356 & & & 879,43 \\
p-76-356 & $-7,96184$ & $-35,625$ & 719,33 \\
p-73-356 & $-7,64961$ & $-35,625$ & 898,61 \\
p-80-353 & $-7,33738$ & $-35,625$ & 1574,61 \\
p-76-353 & $-7,96184$ & $-35,3125$ & 1158,81 \\
p-73-353 & $-7,64961$ & $-35,3125$ & 1246,09 \\
p-80-350 & $-7,33738$ & $-35,3125$ & 2330,54 \\
p-76-350 & $-7,96184$ & -35 & 1565,74 \\
p-73-350 & $-7,64961$ & -35 & 1640,27 \\
\hline
\end{tabular}

Análise de sensibilidade e calibração do modelo

A análise de sensibilidade foi realizada nas sub-bacias 28, 13, 14, 5, e 8 (Figura 3), delimitando assim 5 zonas na bacia do Goiana. Estas áreas foram estabelecidas a partir de estações fluviométricas situadas dentro da bacia.

A vazão observada nestas zonas e utilizada neste trabalho corresponde ao valor observado disponível em cada estação fluviométrica indicada. 
Utilizou-se o software SWAT Calibration and Uncertainty Procedures (SWAT-CUP) para identificar os parâmetros mais sensíveis, passíveis a ajustes para maior coerência em relação aos dados observados e simulados de vazão e realização da posterior calibração. O SWAT-CUP possui uma interface que foi desenvolvida para o SWAT e é capaz de realizar análise de sensibilidade, incertezas e calibração (Abbaspour, 2015).

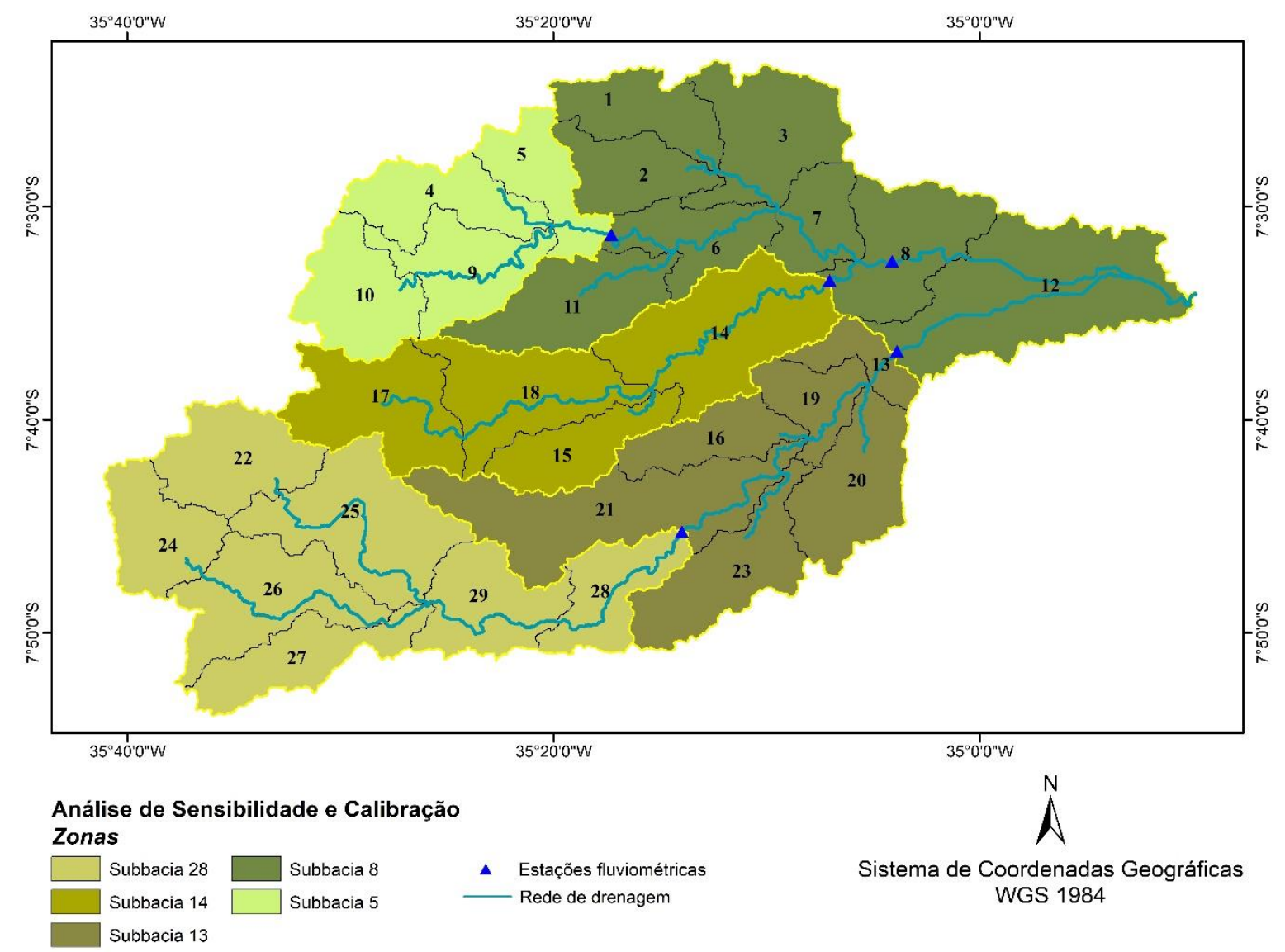

Figura 3. Zonas utilizadas para análise de sensibilidade e calibração espacial.

Existem cinco distintos métodos de calibração no SWAT-CUP, sendo estes: SUFI-2, PSO, GLUE, ParaSol e MSMC. Selecionou-se neste trabalho o método SUFI-2, devido aos bons resultados encontrados em diversos trabalhos (Daguppati et al., 2015a; Pontes et al., 2016; Franco e Bonumá, 2017) e sua aplicabilidade nos desenvolvidos em diversas regiões do Brasil. Este faz uma análise de incerteza e pode trabalhar com uma grande quantidade de parâmetros (Abbaspour, 2005). A predição de $95 \%$ da incerteza (95PPU) é calculada para cada variável simulada (Schuol e Abbaspour, 2006).

Foram elencados alguns parâmetros (Tabela 4) descritos na literatura e o grau de sensibilidade e sua significância foram analisados a partir do t-stat e p-value. Andrade, Mello e Beskow (2013) utilizaram 21 parâmetros e neste trabalho foram elencados 19. Apesar de alguns autores utilizarem um intervalo menor para calibração dos parâmetros, Andrade, Mello e Beskow (2013) trabalharam com limite inferior de
$-50 \%$ e superior de $+50 \%$ para os parâmetros de CN2, SOL_Z, SOL_K e SOL_AWC. Contudo, nesse trabalho optou-se por utilizar um intervalo de variação de menor percentual, de -25 a $+25 \%$ e para o CN2 de apenas -10 e $+10 \%$ como verificado em alguns estudos.

A amostragem dos parâmetros é realizada através do Latin hypercube (Mckay, Beckman e Conover, 2000). A análise de sensibilidade foi realizada de maneira global para as sub-bacias $28,13,14,8$ e 5 , utilizando-se 500 iterações (Abbaspour, 2015; Santos, 2015). A partir da identificação dos parâmetros mais sensíveis, foi procedida a calibração com iterações até encontrar os melhores resultados das estatísticas dentro do intervalo atribuído. As discussões acerca do processo de calibração e validação indicados por Engel et al (2007) e Daggupati et al. (2015b) e foram observadas. O processo de calibração realizado é considerado de estratégia complexa, onde foram utilizadas múltiplas estações (Daggupati et al., 2015b), trabalhando-se a partir 
da upstream para downstream (Arnold et al, 2012). A abordagem sistemática na calibração foi $o$ single-stage que corresponde a análise da resposta do modelo com uma única variável de saída
(Daggupati et al., 2015b), que para este trabalho foi utilizado a vazão. Os melhores valores encontrados foram inseridos através da calibração manual no Arcswat.

Tabela 4. Parâmetros utilizados para análise de sensibilidade no SWAT-CUP

\begin{tabular}{|c|c|c|}
\hline Parâmetros & Descrição & $\begin{array}{l}\text { Intervalos } \\
\text { definidos }\end{array}$ \\
\hline r__CN2.mgt & Curva número na condição 2 de umidade (adimensional) & -0.1 a 0.1 \\
\hline v_ALPHA_BF.gw & Fator de recessão do fluxo de base (dias) & 0 a 1 \\
\hline a_GW_DELAY.gw & Tempo de retardo do fluxo subterrâneo (dias) & -30 a 90 \\
\hline a_GWQMN.gw & $\begin{array}{l}\text { Profundidade mínima do aquífero superficial para escoamento } \\
\text { superficial }\left(\mathrm{mmH}_{2} \mathrm{O}\right)\end{array}$ & -500 a 500 \\
\hline v_ESCO.hru & Fator de compensação da evaporação do solo (adimensional) & 0 a 1 \\
\hline r_SOL_AWC().sol & Capacidade de água disponível ( $\mathrm{mm} \mathrm{H}_{2} \mathrm{O} / \mathrm{mm}$ solo) & -0.25 a 0.25 \\
\hline r_SOL_Z().sol & Profundidade da camada de solo $(\mathrm{mm})$ & -0.25 a 0.25 \\
\hline r__CH_K2.rte & Condutividade hidráulica efetiva no canal principal $(\mathrm{mm} / \mathrm{h})$ & -0.1 a 0.1 \\
\hline v_GW_REVAP.gW & Coeficiente de escoamento subterrâneo (adimensional) & 0.02 a 0.2 \\
\hline v_REVAPMN.gw & Mínimo de água para ocorrência de escoamento superficial (mm) & 0 a 1000 \\
\hline v_RCHRG_DP.gw & Fração de percolação para o aquífero profundo (adimensional) & 0 a 1 \\
\hline r_SLSUBBSN.hru & Comprimento médio do declive (m) & -0.25 a 0.25 \\
\hline r_SOL_K().sol & Condutividade hidráulica $\left(\mathrm{mm} \mathrm{h}^{-1}\right)$ & -0.25 a 0.25 \\
\hline r_USLE_P.mgt & Fator relativo às práticas de conservação do solo & -0.25 a 0.25 \\
\hline r__SOL_ALB().sol & Albedo do solo (adimensional) & -0.25 a 0.25 \\
\hline V_CH_N2.rte & Coeficiente de Manning do canal principal (adimensional) & -0.01 a 0.3 \\
\hline v__CANMX.hru & Armazenamento máximo de água no dossel vegetativo $(\mathrm{mm})$ & 0 a 10 \\
\hline v__BIOMIX.mgt & Eficiência do revolvimento biológico (adimensional) & 0 a 1 \\
\hline v_EPCO.hru & Fator de compensação de água pelas plantas (adimensional) & 0 a 1 \\
\hline v_SURLAG.bsn & $\begin{array}{l}\text { Coeficiente de retardamento do escoamento superficial } \\
\text { (adimensional) }\end{array}$ & 0 a 24 \\
\hline
\end{tabular}

Como procedimentos para calibração, foram utilizados dados mensais de vazão observada correspondente ao período de 1999 a 2009. Os dados foram obtidos a partir de cinco estações fluviométricas adquiridas através do Sistema de Informações Hidrológicas (HIDROWEB) da ANA. As estações Nazaré da Mata, Engenho Itapessirica, Engenho Retiro, Engenho Volta e Caricé foram utilizadas para calibrar as sub-bacias $28,13,14,5$ e 8 respectivamente.

Dentro do período estabelecido para calibração, verificou-se que as estações fluviométricas possuíam lacunas de informação em alguns períodos. Dessa maneira, o percentual de dados sem informação das estações fluviométricas para o período estabelecido foi de $9,8 \%$ para a subbacia $28,6,1 \%$ para a sub-bacia $13,13,6 \%$ para a sub-bacia $14,40,9 \%$ para a sub-bacia 5 e $9,8 \%$ para a sub-bacia 8 .

A calibração foi realizada na seguinte ordem: subbacia 28 , subbasin 13 , subbasin 14 , subbasin 5 e subbacia 8. Após a identificação dos melhores ajustes para cada parâmetro, os ajustes foram realizados nas demais subbacias de cada zona de calibração.

Sabendo-se que é importante analisar os outputs gerados a partir das simulações em relação aos dados observados (Klemes, 1986), a performance do modelo foi analisada segundo múltiplos critérios. Gassman et al. (2007) e Arnold et al. (2012a) apontam que diferentes métodos gráficos e estatísticos podem ser aplicados e dentro os mais comumente utilizados estão o $\mathrm{R}^{2}$, NSE e PBIAS. Nesse estudo utilizou-se a classificação dada por Moriasi et al. (2007) e as estatísticas recomendadas NSE, PBIAS e RSR podem ser representadas pelas seguintes equações:

$$
\begin{aligned}
& N S E=1-\frac{\sum_{i=1}^{n}\left(O_{i}-P_{i}\right)^{2}}{\sum_{i=1}^{n}\left(O_{i}-\bar{O}\right)^{2}} \\
& P B I A S=\frac{\sum_{i=1}^{n}\left(O_{i}-P_{i}\right)}{\sum_{i=1}^{n} O_{i}} \times 100 \% \\
& R S R=\frac{\sqrt{\sum_{i=1}^{n}\left(O_{i}-P_{i}\right)^{2}}}{\sqrt{\sum_{i=1}^{n}\left(O_{i}-\bar{O}\right)^{2}}}
\end{aligned}
$$


Onde $O_{i}$ se refere ao dado observado, $\mathrm{P}$ ao resultado após a modelagem, $\bar{O}$ a média dos dados observados e n é o número de observações.

\section{Resultados e discussão}

\section{Análise de sensibilidade}

Devido a extensão do território brasileiro e as diferenças encontradas nas cinco regiões, a parametrização das bacias hidrográficas pode apresentar diferenças entre estudos brasileiros, contudo alguns parâmetros podem ser destacados pelo alto índice de sensibilidade. A Tabela 5 apresenta o resultado da análise de sensibilidade automática realizada pelo SWAT CUP, onde verifica-se quais os parâmetros mostraram-se mais sensíveis a calibração da vazão. Alguns desses parâmetros considerados mais sensíveis também foram identificados em outros trabalhos.

Verifica-se que dos 20 parâmetros utilizados na análise para vazão, nove apresentaram as maiores sensibilidades, sendo estes o CN2, GW_DELAY, ESCO, SOL_AWC, SOL_Z, RCHRG_DP, USLE_P, CANMX e EPCO. Já no trabalho de Me, Abell e Hamilton (2015), os autores apontaram 21 parâmetros sensíveis que foram calibrados para vazão, modificando diversos parâmetros da modelagem inicial.

Os parâmetros que apresentaram maior sensibilidade no presente trabalho se referem a manejo de culturas/vegetação, solo, e água subterrânea. Isso se dá, pois, conforme o trabalho de Schmalz e Fohrer (2009), os parâmetros de maior influência na modelagem hidrológica são os relacionados a água subterrânea e solo. Devi, Ganasri e Dwarakish (2015) ainda colocam que os dados meteorológicos e as propriedades do solo correspondem a parâmetros de influência na performance dos modelos. Isso se dá devido aos dados e condições meteorológicas influenciarem nas condições de vegetação, solo e água subterrânea e escoamento superficial.

Como a análise de sensibilidade foi diferenciada para as distintas bacias, verifica-se que conforme Aragão et al. (2013) os processos ocorridos nas bacias são diferentes e os mesmos parâmetros não devem ser utilizados para calibração de distintas bacias. E que de acordo com Lelis et al. (2012), o tamanho das sub-bacias não influencia na sensibilidade dos parâmetros.

No levantamento realizado por Arnold et al. (2012) foi evidenciado que muitos trabalhos utilizam CN2, SOL_AWC, ESCO, GW_ALPHA e
SURLAG nos procedimentos de calibração, o que indica a sensibilidade desses. Para Feyereisen et al. (2007) os parâmetros que apresentaram maior sensibilidade relativa para produção de água, vazão de tempestade e vazão de base foram referentes a respostas na superfície como CN2, SOL_AWC, ESCO e SOL_BD. Schuol e Abbaspour (2006) encontraram CN2, SOL_AWC, ESCO, SURLAG, REVAPMN, RCHEG_DP, e MSK. Destes parâmetros o SURLAG não apresentou sensibilidade para nenhuma das sub-bacias do Goiana, apesar da verificação da sensibilidade em outros trabalhos. Isso se dá pelas diferenças nas características físicas e climáticas das bacias, que influenciam nos valores dos parâmetros e assim na sua análise de sensibilidade. Além disso, o uso e ocupação de cada sub-bacia também tem influência nos resultados, conforme Lelis et al. (2012).

Para Nossent, Elsen e Bauwens (2011) os parâmetros mais sensíveis foram referentes a $\mathrm{CN} 2$, CH_N e GWQMN para calibração da vazão. Pereira et al. (2016) trabalhando na bacia do Rio Pomba no sudeste brasileiro identificou SOL_K, APLHA_BF, ESCO, CN2, CH_N2, SOL_AWC e SOL_Z como mais sensíveis. Para Andrade, Mello e Beskow (2013) os parâmetros encontrados para a bacia do Ribeirão Jaguara, na região Alto Rio Grande em Minas Gerais, foram CN2, ALPHA_BF, RCHRG_DP, ESCO, SOL_Z, SOL_K, SOL_AWC.

Fukunaga et al. (2015) encontraram como parâmetros sensíveis os relacionados a solo, uso e cobertura do solo, águas subterrâneas e rede de transmissão, como CN2, GWQMN, ESCO, CANMIX, SOL_K, SOL_AWC, SOL_Z, ALPHA_BF, BLAI, CH_K2, CH_N2, GW_DELAY, SOL_ALB, EPCO, REVAPMN e SURLAG. Contudo, os autores optaram por não utilizar todos os parâmetros na calibração.

O ALPHA_BF que é descrito muitas vezes como um parâmetro sensível em alguns trabalhos, teve uma das menores influências. Este parâmetro tem sido relatado em diversos trabalhos como $\mathrm{Wu}$ e Johnston (2007), Andrade, Mello e Beskow (2013), Bressiani et al. (2015), Fukunaga et al. (2015), contudo teve uma das menores influências e não foi utilizado na calibração do presente estudo devido possuir uma sensibilidade inferior aos demais parâmetros analisados.

Daggupati et al. (2015b) mencionaram que nem todos os parâmetros considerados sensíveis necessitam ser calibrados, isso pode ser avaliado a partir da experiência do usuário em modelagens ou a partir de indicações da literatura. Isso aconteceu no presente trabalho onde o EPCO, apesar de se apresentar como um parâmetro sensível, não foi 
Revista Brasileira de Geografia Física v.11, n.1, (2018) 371-389.

utilizado na calibração. Este é geralmente aplicável quando se verifica a existência de grande fragmentação de diferentes usos de solo na bacia.

Tabela 5. Análise de sensibilidade dos parâmetros para vazão

\begin{tabular}{llllll}
\hline Parâmetros & SB 28 & SB 13 & SB 14 & SB 5 & SB 8 \\
\hline r__CN2.mgt & 1 & 14 & 1 & 1 & 8 \\
v_ALPHA_BF.gw & 18 & 19 & 17 & 15 & 20 \\
a__GW_DELAY.gw & 6 & 7 & 6 & 4 & 3 \\
a_GWQMN.gw & 16 & 18 & 14 & 6 & 18 \\
v__ESCO.hru & 2 & 2 & 2 & 5 & 2 \\
r__SOL_AWC().sol & 4 & 3 & 7 & 3 & 5 \\
r__SOL_Z().sol & 5 & 6 & 10 & 7 & 6 \\
r__CH_K2.rte & 19 & 17 & 20 & 17 & 15 \\
v_GW_REVAP.gw & 17 & 5 & 5 & 13 & 13 \\
v_REVAPMN.gw & 14 & 12 & 12 & 14 & 11 \\
v__RCHRG_DP.gw & 3 & 1 & 3 & 18 & 1 \\
r__SLSUBBSN.hru & 10 & 15 & 15 & 10 & 17 \\
r__SOL_K().sol & 9 & 11 & 13 & 11 & 14 \\
r__USLE_P.mgt & 12 & 9 & 11 & 9 & 9 \\
r__SOL_ALB().sol & 11 & 13 & 16 & 8 & 12 \\
v_CH_N2.rte & 13 & 10 & 8 & 12 & 10 \\
v_CANMX.hru & 7 & 8 & 9 & 6 & 7 \\
v_BIOMIX.mgt & 15 & 16 & 19 & 19 & 19 \\
V__SURLAG.bsn & 20 & 20 & 18 & 20 & 16 \\
v_EPCO.hru & 8 & 4 & 4 & 2 & 4 \\
\hline
\end{tabular}

\section{Calibração do modelo}

Com base nos parâmetros obtidos na análise de sensibilidade, os mais sensíveis para cada uma das sub-bacias sofreram ajustes de valores através do processo de calibração da vazão. Para cada uma das sub-bacias verifica-se que os valores encontrados foram distintos, ainda que se tenha trabalhado com os mesmos parâmetros em distintas sub-bacias, conforme pode-se verificar na Tabela 6 .

Tabela 6. Parâmetros utilizados na calibração

\begin{tabular}{llllll}
\hline $\begin{array}{l}\text { Parâmetros } \\
\text { subbacia 28 }\end{array}$ & $\begin{array}{l}\text { Valor } \\
\text { calibrado }\end{array}$ & $\begin{array}{l}\text { Parâmetros } \\
\text { subbacia 13 }\end{array}$ & $\begin{array}{l}\text { Valor } \\
\text { calibrado }\end{array}$ & $\begin{array}{l}\text { Parâmetros } \\
\text { subbacia 14 }\end{array}$ & $\begin{array}{l}\text { Valor } \\
\text { calibrado }\end{array}$ \\
\hline CN2 & -0.08519 & RCHRG_DP & 0.381004 & CN2 & -0.07262 \\
RCHRG_DP & 0.471794 & ESCO & 0.276827 & ESCO & 0.438359 \\
ESCO & 0.303912 & SOL_AWC & 0.248508 & RCHRG_DP & 0.455415 \\
SOL_AWC & 0.248123 & GW_REVAP & 0.087755 & GW_REVAP & 0.130872 \\
SOL_Z & 0.199801 & SOL_Z & 0.095000 & GW_DELAY & -27.52166 \\
GW_DELAY & -24.1585 & GW_DELAY & -29.9764 & SOL_AWC & 0.104324 \\
CANMX & 19.26619 & CANMX & 4.608878 & CH_N2 & 0.277646 \\
SOL_K & -0.14453 & USLE_P & -0.06060 & CANMX & 9.532859 \\
SLSUBBSN & 0.014511 & - & - & SOL_Z & 0.031403 \\
\hline
\end{tabular}


Revista Brasileira de Geografia Física v.11, n.1, (2018) 371-389.

Tabela 6. (Continuação) Parâmetros utilizados na calibração

\begin{tabular}{lllc}
\hline $\begin{array}{l}\text { Parâmetros } \\
\text { subbacia 5 }\end{array}$ & $\begin{array}{l}\text { Valor } \\
\text { calibrado }\end{array}$ & $\begin{array}{l}\text { Parâmetros } \\
\text { subbacia 8 }\end{array}$ & $\begin{array}{l}\text { Valor } \\
\text { calibrado }\end{array}$ \\
\hline CN2 & -0.09295 & CN2 & -0.00789 \\
SOL_AWC & 0.208182 & GW_DELAY & -7.28545 \\
GW_DELAY & 53.05331 & ESCO & 0.501378 \\
ESCO & 0.672376 & SOL_AWC & 0.092922 \\
GWQMN & 990.3423 & SOL_Z & 0.254504 \\
SOL_ALB & 0.231693 & REVAPMN & 596.7773 \\
USLE_P & 0.071065 & RCHRG_DP & 0.021149 \\
SLSUBBSN & -0.21433 & USLE_P & -0.00640 \\
SOL_K & 0.213173 & CH_N2 & 0.170758 \\
& & CANMX & 8.472565 \\
\hline
\end{tabular}

Para as diferentes sub-bacias calibradas nesse estudo, os valores finais dos parâmetros obtidos (Tabela 9) foram comparados com valores utilizados por outros trabalhos brasileiros e internacionais, analisando as características climáticas dos locais. Para o ESCO, baseado em alguns estudos, Wu e Johnston (2007) indicaram que para condições climáticas médias o valor é 0,5 e para condições mais secas 0,8 . Para o presente trabalho, o parâmetro foi sensível a todas as subbacias utilizadas no processo de calibração e o maior e menor valor encontrado foram respectivamente para as sub-bacias 5 e 13 de 0,67 e 0,28 .

O valor calibrado para ESCO por Pereira et al. (2016) foi de 0,3 para uma bacia no sudeste brasileiro que possui média anual de precipitação de $1400 \mathrm{~mm}$. Valor este similar ao calibrado para a sub-bacia 28. Bressiani et al. (2015) apontou como melhor valor 0,6 para a bacia do Jaguaribe localizada numa área semiárida do nordeste brasileiro. Valor este que se aproxima do valor encontrado para a sub-bacia 5, cuja pluviosidade anual é inferior as demais sub-bacias. Castro et al. (2013) obteve 0,1 para a bacia experimental Alto Jardim localizada no do cerrado brasileiro no Distrito Federal. Um valor similar também foi obtido por Kim, Kim e Kang (2016) para uma bacia localizada na Coréia do Sul. Shuol e Abbaspour (2006) apresentaram um intervalo final de calibração que variou de 0,12 a 0,50 para uma robusta bacia no oeste da África que inclui a bacia do rio Niger, Volta e Senegal. Fukunaga et al. (2015) encontrou um valor para calibração de 0,566 para uma bacia localizada no Espírito Santo. Andrade, Mello e Beskow (2013) encontrou valor inferior para este parâmetro $(0,043)$ para uma área com temperatura média anual de $19^{\circ} \mathrm{C}$ e precipitação anual de $1500 \mathrm{~mm}$, na região Alto Rio Grande em Minas Gerais.

Para o parâmetro referente a fração de percolação no aquífero profundo RCHRG_DP, alguns autores apontam os valores modificados após o processo de calibração. Estes foram 0,1 para Bressiani et al. (2015), 0.484 para Andrade, Mello e Beskow (2013). O intervalo utilizado por Shuol e Abbaspour (2006) variou de 0,56 a 0,70. Neste trabalho esse parâmetro se mostrou sensível para as sub-bacias $28,13,14$ e 8 , tendo obtido diferentes valores durante o processo de calibração, sendo respectivamente $0,47,0,38,0,45$ e 0,02 . Os distintos valores referentes a esse parâmetro estão relacionados ao tipo de solo e ao uso e cobertura da área da sub-bacia e condições climáticas. Áreas com fragmentos de Mata Atlântica são fundamentais na recarga de água subterrânea, como identificado no trabalho de Alvarenga et al. (2012).

O SOL_AWC também foi considerado como sensível em alguns trabalhos e Shuol e Abbaspour (2006) indicaram o intervalo utilizado na calibração de 0,145 a 0,175 . Este parâmetro foi considerado sensível para todas as sub-bacias analisadas e o seu percentual de variação foi de $0,25,0,21,0,25,0,10$ e 0,09 respectivamente para as bacias $28,5,13,14$ e 8 . A capacidade de água disponível pode ser descrita como a capacidade que um solo tem de armazenar e liberar água para as raízes das plantas (Silva et al., 2014) e para todas as bacias, os ajustes realizados foram com percentuais positivos. As variações dos valores são referentes aos diferentes tipos de solo $\mathrm{e}$ as condições modeladas para cada uma das subbacias. Apesar da modificação nesse parâmetro ser em percentual, não substituindo valores anteriormente definidos no modelo, deve-se ter cuidado ao utilizar valores muito altos para não forçar condições naturais apenas para alcance de estatísticas superiores. Fukunaga et al. (2015) acrescentou $41,8 \%$ a este parâmetro no processo de calibração, contudo nesse trabalho limitou-se aos $25 \%$.

A água da chuva pode ser interceptada pela vegetação e atingir o solo posteriormente, 
influenciando em aspectos como infiltração da água no solo e escoamento superficial. Tendo, portanto, importância nos estudos hidrológicos (Xiau et al., 2000). A parcela de água interceptada é diferente para cada tipo de vegetação e com relação a CANMIX, Fukunaga et al. (2015), para uma região com vegetação agrícola calibrou o valor em $11.3 \mathrm{~mm}$, onde há plantação de café, eucalipto, pastagem, floresta nativa, floresta de regeneração secundária, área urbana e pedras. Pereira et al. (2014a) realizou trabalho em uma área de Mata Atlântica, mas não realizou alteração no parâmetro CANMIX, modificando-se outros parâmetros de vegetação do SWAT como BLAI, GSI, RDMX e OV_N. Neste trabalho alterou-se os valores de CANMIX para 19,27, 4,60, 9,53 e $8,47 \mathrm{~mm}$ respectivamente para as sub-bacias 28,13 , 14 e 8 .

Em relação aos parâmetros de águas subterrâneas, Fukunaga et al. (2015) alteraram GW_DELAY para 287 dias, GW_REPVAP para 0,188 , GWQMN para $3907 \mathrm{~mm}$. Nas sub-bacias do presente estudo, foram subtraídos valores do GW_DELAY nas sub-bacias 28 com -24,16 dias, na sub-bacia 13 com -29,98 dias, na sub-bacia 14 com -27,52 dias e na sub-bacia 8 com -7,29 dias. Somente na sub-bacia 5 que foi acrescido o valor de 53,05 dias devido consistir em uma região de climatologia mais seca que as demais. $\mathrm{O}$ atraso relacionado ao tempo em que a água sai do perfil do solo e alcança o aquífero pode ser relacionado as propriedades hidráulicas das formações geológicas das áreas (Arnold et al., 2012b) e por isso difere para cada localidade da bacia. Além disso, outras condições hidrogeológicas também contribuem para o fluxo da água subterrânea, como transmissividade e coeficiente de armazenamento Tabela 7. Comparativo das simulações antes e após calibração para estações locais e globais

\begin{tabular}{llllllll}
\multirow{2}{*}{ Sub-bacia } & \multicolumn{3}{c}{ Estações locais (L) } & \multicolumn{3}{c}{ Estações L+G } \\
& NSE & PBIAS & RSR & NSE & PBIAS & RSR \\
\hline 28I & 0,47 & $-82,58$ & 0,73 & 0,56 & $-75,74$ & 0,66 \\
28II & 0,79 & $-10,69$ & 0,46 & 0,80 & $-6,76$ & 0,44 \\
13I & $-0,19$ & $-116,0$ & 1,01 & $-0,09$ & $-112,3$ & 1,04 \\
13II & 0,80 & $-21,53$ & 0,44 & 0,87 & $-17,00$ & 0,36 \\
14I & $-0,23$ & $-67,08$ & 1,11 & 0,05 & $-39,27$ & 0,98 \\
14II & 0,62 & 4,43 & 0,62 & 0,34 & 30,08 & 0,80 \\
5I & 0,08 & $-18,08$ & 0,71 & 0,08 & $-18,08$ & 0,71 \\
5II & 0,43 & 17,99 & 0,56 & 0,43 & 17,99 & 0,56 \\
8I & 0,46 & $-61,69$ & 0,73 & 0,58 & $-41,43$ & 0,65 \\
8II & 0,82 & $-9,70$ & 0,42 & 0,80 & 11,62 & 0,44 \\
\hline
\end{tabular}

I - Sem calibração; II - Após calibração ${ }^{1}$ indicados no trabalho de Tirogo et al. (2016) em Burkina Faso no Oeste da África.

As estatísticas encontradas (Tabela 7) demostram uma melhoria nos resultados após a realização da etapa de calibração. Os resultados indicam que o uso de estações observadas (locais) acrescidos de dados de reanálise (globais) trouxe resultados mais satisfatórios para algumas áreas da bacia do Goiana, devido a uma melhor distribuição de informações de precipitação ao longo da bacia. Contudo, para a sub-bacia 8 as estatísticas apresentaram leves reduções na qualidade da representação da simulação e na sub-bacia 14 houve uma piora, apresentando resultados insatisfatórios. Isso se dá devido a uma introdução de dados que não representam a mesma realidade climática da sub-bacia, alterando suas saídas.

As sub-bacias que apresentaram simulações com resultados mais próximos do observado foram a sub-bacia 8 com NSE de 0,82 , PBIAS -9,70 e RSR 0,42 e a sub-bacia 28 com NSE 0,79, PBIAS -10,69 e RSR 0,46 para dados de estações locais. Já para simulações a partir de estações locais+globais, as mesmas estações apresentaram os melhores resultados, sub-bacia 8 com NSE de 0,80, PBIAS 11,62 e RSR 0,44 e a sub-bacia 28 com NSE 0,80 , PBIAS -6,76 e RSR 0,44 .

Contudo, a sub-bacia 5 apresentou resultados insatisfatórios para ambas as simulações, devido a consistir de uma área da bacia onde os dados climáticos são mais escassos e menos distribuídos. Além desses aspectos, essa área da bacia também possui uma climatologia um pouco mais seca que as demais áreas e tem os valores mais baixos de vazão observada (Figura 4

\footnotetext{
1 Após o procedimento de calibração, utilizando-se dados climáticos locais, a performance alcançada utilizando a classificação de Moriasi et al. (2007) foi de: SB28 - boa; SB13 - satisfatória; SB14 - satisfatória; SB5 - insatisfatória; e SB8 - muito boa. Para dados climáticos locais combinados a dados globais as performances obtidas foram: SB28 - muito boa; SB13 - satisfatória; SB14 - insatisfatória; SB5 - insatisfatória; SB8 - boa.
} 

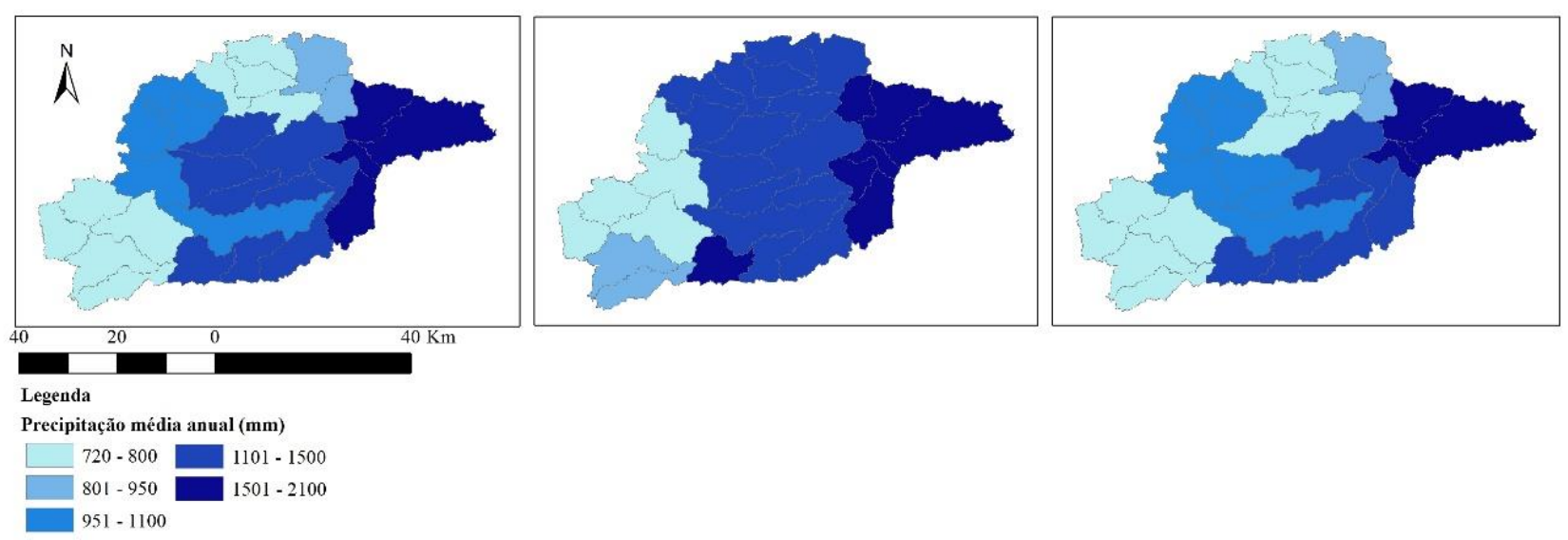

Figura 4. Precipitação média anual da bacia no período de 1999 a 2009 utilizando dados climáticos (a) observados locais, (b) de reanálise globais e (c) ambos.

Apesar da obtenção de resultados satisfatórios se utilizando os dados climáticos locais+globais, o resultado insatisfatório encontrado na sub-bacia 14 aponta para a importância da realização de testes com dados de reanálise, para verificar se as características climáticas desses dados representam a realidade local.

Em outros trabalhos brasileiros utilizandose o SWAT, estatísticas similares foram encontradas como o de Brighenti et al. (2016) encontrou valores de 0,70 e 0,73 para distintos períodos de calibração e 0,63 e 0,55 para validação de vazões mensais na bacia do Rio Negrinho em Santa Catarina com área aproximada de $200 \mathrm{~km}^{2}$. Pereira et al. (2016) para dados diários de vazão alcançou um NSE adequado para a calibração e validação de 0,76 e PBIAS de 4,6 e 5,1 respectivamente para uma bacia de $8600 \mathrm{~km}^{2}$ no sudeste brasileiro. O resultado encontrado por Andrade, Mello e Beskow (2013) também foi satisfatório, obtendo NSE de 0,66 e 0,87, PBIAS de 4,33 e -1,59 nas etapas de calibração e validação do modelo na bacia que possui $32 \mathrm{~km}^{2}$. Pereira et al. (2014a) encontraram NSE de 0,65 e 0,70 para os períodos de calibração e validação de dados diários de vazão na bacia do Córrego do Galo no Espírito Santo, possibilitando a simulação de efeitos para distintos cenários de preservação e degradação ambiental. Castro et al. (2013) encontrou o NSE de 0,66 para calibração diária numa bacia do cerrado brasileiro. Fukunaga et al. (2014) também encontrou valores satisfatórios para calibração diária, obtendo NSE de 0,75, PBIAS de 11 e RSR de 0,50 . Durante a validação os valores obtidos foram $0,67,22$ e 0,57 .
Apesar de algumas sub-bacias do presente trabalho terem apresentando resultados muito bons ou bons, de acordo com a classificação de Moriasi et al. (2007), verifica-se que os picos dos gráficos não são alcançados em todos os meses nas simulações (Figura 5). Contudo, é importante salientar que as sub-bacias com melhores estatísticas possuem um ajuste mais adequado das curvas de vazão. A curva da sub-bacia 5 possui diferenças entre os picos e deslocamento posterior ou atrasos. Durante os períodos de vazão mais secos, onde a vazão se encontra mais baixa, também se percebe uma variação entre os valores simulados e os observados.

Questões como essas em relação a períodos chuvosos e secos foram discutidas em alguns trabalhos como Feyereisen et al. (2007) que verificaram que o modelo trabalhado na bacia experimental de Little River no sudoeste da Geórgia gerava melhores resultados para anos mais úmidos que o normal que para anos mais secos que o normal. A sub-bacia 5 além de apresentar uma climatologia mais seca que as demais áreas da bacia, apresenta vazões inferiores durante todo o período analisado, com valores mínimos de zero e valores máximos próximos a $25 \mathrm{~m}^{3} / \mathrm{s}$.

Feyereisen et al. (2007) encontraram para produção de água um NSE de 0,89 para modelagem mensal e 0,55 para diária nos anos úmidos, em contrapartida que nos anos secos eles obtiveram 0,59 para modelagem mensal e 0,22 para diária. Já para simulação de vazão, os valores também tiveram a mesma resposta para o ano úmido um NSE diário de 0,62 e para o ano seco NSE diário de $-0,80$. Na modelagem da sub-bacia 
5 para vazão mensal, após a calibração o resultado foi de NSE 0,43 e PBIAS 17,99.

Isto também foi constatado por Van Liew et al. (2007) que testou a performance do SWAT em cinco bacias experimentais da USDA ARS nos Estados Unidos verificando melhor desempenho para regiões úmidas que áreas desérticas ou semidesérticas. Brighenti et al. (2016) realizando a calibração individual para cada ano entre 2003 e 2012, verificaram calibração e validação mais eficientes em anos úmidos que em anos secos. Em contraponto, Govender e Everson (2005) verificaram que a performance do modelo foi superior em anos secos que anos úmidos em uma bacia experimental no sul da África.

Para este trabalho, as áreas úmidas da bacia apresentaram resultados superiores tanto estatisticamente quanto graficamente, corroborando com os trabalhos desenvolvidos por outros pesquisadores que alcançaram respostas similares.

\section{Conclusões}

A análise de sensibilidade realizada através do SWATCUP proporcionou a identificação de parâmetros mais sensíveis e uma calibração direcionada a estes parâmetros elencados. Dos parâmetros utilizados na análise, os que apresentaram as maiores sensibilidades foram o CN2, GW_DELAY, ESCO, SOL_AWC, SOL_Z, RCHRG_DP, USLE_P, CANMX e EPCO.

Verificou-se também que apesar de ser uma única bacia e de dimensão média, por possuir distintos usos do solo, e regiões com características climáticas distintas, os parâmetros de sensibilidade foram diferentes, assim como os valores obtidos após a calibração; indicando-se, portanto, uma calibração espacializada.

Pôde-se verificar que o uso de dados observados de estações locais garantiu um maior número de estações satisfatoriamente calibradas. Contudo, fazendo-se uso de dados climáticos locais mais globais houve uma melhora das estatísticas de sub-bacias cujas estações pluviométricas estavam melhores distribuídas.

Os resultados alcançados demonstram a possibilidade do uso de dados observados e de reanálise juntos onde exista deficiência de informações ou estações com grande número de falhas, contudo deve-se observar que em áreas com má distribuição de estações e regiões mais secas podem não fornecer respostas adequadas.

As estatísticas alcançadas na modelagem desenvolvida no SWAT evidenciam a aplicabilidade do modelo para estimativas mensais de vazão. Este demostrou boas predições para a sub-bacia 8, sub-bacia com dados observados disponíveis localizada mais próximo ao exutório principal, que recebe a vazão da zona em que está localizada um sub-bacia 5 e da zona onde está localizada uma sub-bacia 14. contudo verificam-se restrições para a área mais seca da bacia, sub-bacia 5 , cujos resultados foram insatisfatórios.

\section{Agradecimentos}

Os autores agradecem a Capes pela concessão de bolsa de doutorado no país e doutorado sanduíche no exterior pelo projeto PVE 103/2013 ao primeiro autor. A Texas A\&M University pela orientação e execução da pesquisa no exterior. Ao CNPq pelo projeto 446254/2015-0. 
Revista Brasileira de Geografia Física v.11, n.1, (2018) 371-389.
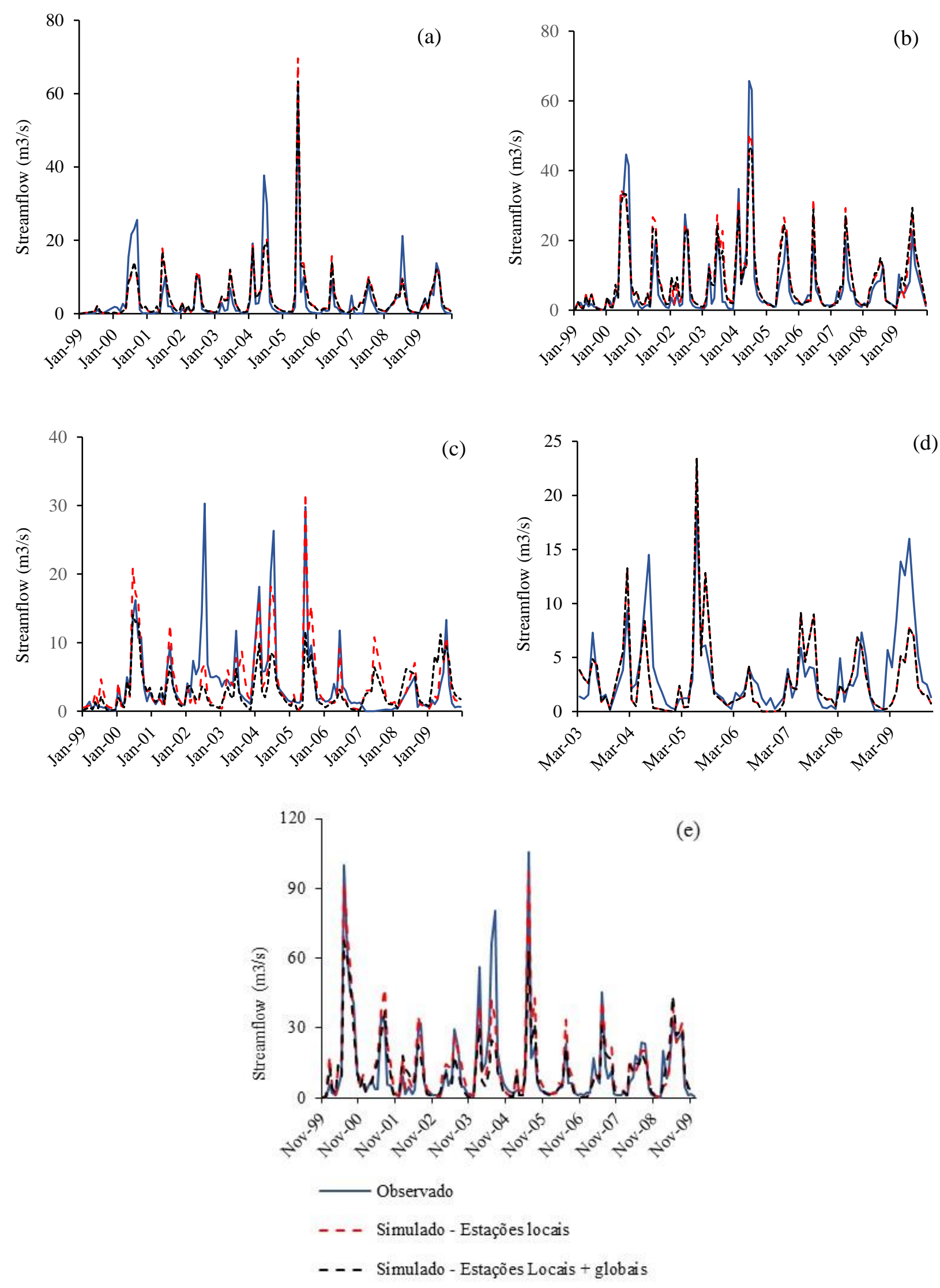

Figura 5. Comparativo das calibrações realizadas nas sub-bacia 28 (a), sub-bacia 13 (b), sub-bacia 14 (c), subbacia 8 (d) e sub-bacia 5 (e) para o período de 1999 a 2009 utilizando dados climáticos locais e globais mensais. 


\section{Referências}

Abbaspour, K.C., 2005. Calibration of Hydrologic Models: When is a Model Calibrated?I n: Proc. Intl. Congress on Modelling and Simulation (MODSIM'05), 2449-2455.

Abbaspour, K.C., 2015. Swat-Cup: SWAT Calibration and Uncertainty Programs Manual. Eawag. Swiss Federal Institute of Aquatic Science and Technology, Duebendorf, Switzerland.

Alvarenga, C.C., Mello, C.R., Mello, J.M., Silva, A.M., Curi, N., 2012. Índice de qualidade do solo associado à recarga de água subterrânea (IQSRA) na bacia hidrográfica do Alto Rio Grande, MG. R. Bras. Ci. Solo 36, 1608-1619.

Aragão, R., Cruz, M.A.S., Amorim, J.RA., Mendonça, L.C., Figueiredo, E. E., Srinivasan, V.S., 2013. Análise de sensibilidade dos parâmetros do modelo SWAT e simulação dos processos hidrossedimentológicos em uma bacia no agreste nordestino. Rev. Bras. Ciênc. Solo, 37, 4, 10911102. DOI: $10.1590 / \mathrm{S} 0100-06832013000400026$

Arnold, J.G., Allen, P.M., Volk, M., Williams, J.R., Bosch, S.D., 2010. Assessment of different representations of spatial variability on SWAT model performance. Transactions of the ASABE $53,1433-1443$.

Arnold, J.G., Srinivasan, R., Muttiah, R.S.,Williams, J.R., 1998. Large area hydrologic modeling and assessment. Part I: model development. Journal of the American Water Resources Association 34, 73-89. DOI: 10.1111/j.1752-1688.1998.tb05961.x

Arnold, J.G., Moriasi, D.N., Gassman, P.W., Abbaspour, K.C, White, M.J., Srinivasan, R., Santhi, C., Harmel, R.D., Van Griensven, A., Van Liew, M.W., Kannan, N., Jha, M.K., 2012a. Swat: model use, calibration, and validation. Transactions of the ASABE 55, p. 1491-1508.

Arnold, J.G., Kiniry, J.R., Srinivasan, R., Williams, J.R., Haney, E.B., Neitsch, S.L. 2012b. Input/Output Documentation. Texas Water Resources Institute.

Andrade, M. A., Mello, C. R. De., Beskow, S., 2013. Simulação hidrológica em uma bacia hidrográfica representativa dos Latossolos na região Alto Rio Grande, MG. Revista brasileira de engenharia agrícola e ambiental 17, 1, p.69-76.

APAC. Bacias Hidrográficas. Rio Goiana. Disponível em:

$<$ http://www.apac.pe.gov.br/pagina.php?page_id= 5\&subpage_id=15> Acesso: 29 nov. 2016.

Blainski, E., Acosta, E., Nogueira, P. C.P., 2017. Calibração e validação do modelo SWAT para simulação hidrológica em uma bacia hidrográfica do litoral norte catarinense. Revista Ambiente e Água 12, 2, 226-237.

Bressiani, D. A., Srinivasan, R., Jones, C.A., Mendiondo, E.M., 2015. Effects of spactial and temporal weather data resolutions on streamflow modeling a semi-arid basin, Northeast Brasil. Int J. Agric. \& Biol. Eng. 8, 125-139. DOI: 10.3965/j.ijabe.20150803.970

Brighenti, T.M., Bonuma, N.B., Chaffe, P.L.B., 2016. Calibração hierárquica do modelo swat em uma bacia hidrográfica Catarinense. Revista Brasileira de Recursos Hidricos 21, 1, 53-64.

Castro, K.B., 2013. Avaliação do modelo SWAT na simulação da vazão em bacia agrícola do cerrado intensamente monitorada. Dissertação (Mestrado). Universidade de Brasília.

CONDEPE/FIDEM, 2005. Agência Estadual de Planejamento e Pesquisas de Pernambuco. Rio Goiana e GL 6. Recife.

Cotter, A.S., Chaubey, I., Costello, T., Soerens, T.S., Nelson, M.A., 2003. Water quality model output uncertainty as affected by spatial resolution of input data. Journal of the American Water Resources Association 39, 977-986. DOI: 10.1111/j.1752-1688.2003.tb04420.x

CPRH. Agência Estadual de Meio Ambiente, 2003. Diagnóstico Socioambiental do Litoral Norte de Pernambuco. Recife: CPRH.

Daggupati, P., Yen, H., White, M., Srinivasan, R., Arnold, J.G., Keitzer, C.S., Sowa, S.P., 2015a. Impact of model development, calibration and validation decisions on hydrological simulations in 
West Lake Erie Basin. Hydrological Processes 29, 5307-5320. DOI: 10.1002/hyp.10536

Daggupati, P., Pai, N., Douglas-Mankin, K.R., Zeckoski, R.W., Jeong, J., Parajuli, P.B., Saraswat, D., Youssef, M.A., 2015b. A recommended calibration and validation strategy for hydrologic and water quality models. Transactions of the ASABE 58, 1705-1719. DOI: $10.13031 /$ trans.58.10712

Devi, G.K, Ganasri, B.P., Dwarakish, G.S., 2015. A Review on Hydrological Models. Aquatic Procedia 4, 1001-1007. DOI: 10.1016/j.aqpro.2015.02.126

Dile, Y.T., Srinivasan, R., 2014. Evaluation of CFSR Climate Data for Hydrologic Prediction in DataScarce Watersheds: An Application in the Blue Nile River Basin. Journal of the American Water Resources Association (JAWRA) 50, 1-16. DOI: 10.1111/jawr.12182

Eduardo, E.N., Mello, C.R., Viola, M.R., Owens, P.R., Curi, N., 2016. Hydrological simulation as subside for management of surface water resources at the Mortes River Basin. Ciênc. agrotec. 40, 390404. DOI: $10.1590 / 1413-70542016404009516$

El-Sadek, A., Bleiweiss, M., Shukla, M., Guldan, S., Fernald, A., 2011. Alternative climate data sources for distributed hydrological modelling on a daily time step. Hydrological Processes 25, 1542-1557. DOI: 10.1002/hyp.7917

EMBRAPA. Empresa Brasileira de Pesquisa Agropecuária, 2013. Sistema Brasileiro de Classificação de Solos. SANTOS, H. G. et al. 3 ed. Embrapa, Brasília.

Engel, B., Storm, D., White, M., Arnold, J., Arabi, M., 2007. Hydrologic Water Quality Model Application Protocol. Journal of the American Water Resources Association (JAWRA) 43, 12231236. DOI: 10.1111/j.1752-1688.2007.00105.x

Feyereisen, G.W., Strickland, T.C., Bosch, D.D, Sullivan, D.G., 2007. Evaluation of SWAT manual calibration and input parameter sensitivity in the little river watershed. Transactions of the ASABE 50, p. 843-855.
Franco, A.C.L., Bonumá, N.B., 2017. Multi-variable SWAT model calibration with remotely sensed evapotranspiration and observed flow. RBRH 2, e35. DOI: 10.1590/2318-0331.011716090

Fuka, D.R., Walter, M.T., MacAlister, C., Degaetano, A.T., Steenhuis, T.S., Easton, Z., 2014. Using the Climate Forecast System Reanalysis as weather input data for watershed models. Hydrological Processes 28, 5613-5623. DOI: 10.1002/hyp.10073

Fukunaga, D.C., Cecílio, R.A., Zanetti, S.S., Oliveira, L.T., Caiado, M.A.C., 2015. Application of the SWAT hydrologic model to a tropical watershed at Brazil. Catena 125, 206-213. DOI: 10.1016/j.catena.2014.10.032

Gassman, P.W., Reyes, M.R., Green, C.H., Arnold, J.G., 2007. The soil and water assessment tool: historical development, applications, and future research directions. Transactions of the ASABE 50, 1211-1250.

Govender, M., Everson, C.S., 2005. Modelling streamflow from two small South African experimental catchments using the SWAT model. Hydrological processes 19, 683- 692. DOI: 10.1002/hyp.5621

IESB. Instituto de Estudos Socioambientais do Sul da Bahia, 2007. Levantamento da Cobertura Vegetal Nativa do Bioma Mata Atlântica. Projeto de conservação e utilização sustentável da diversidade biológica brasileira - PROBIO. Edital PROBIO 03/2004. Rio de Janeiro.

Kim, Y., Kim, M.J., Kang, B., 2016. Projection of runoff and sediment yield under coordinated climate change and urbanization scenarios in Doam Dam watershed, Korea. Journal of Water and Climate Change. DOI: 10.2166/wcc.2016.068

Klemes, V., 1986. Operational testing of hydrological simulation models. Hydrological Sciences Journal $3,13-24$.

Lelis, T.A., Calijuri, M.L., Santiago, A.F., Lima, D.C., Rocha, E.O., 2012. Análise de sensibilidade e calibração do modelo SWAT aplicado em bacia hidrográfica da região sudeste do Brasil. Revista Brasileira de Ciência do Solo 36, 623-634. DOI: 10.1590/S0100-06832012000200031. 
Mckay, M.D., Beckman, R.J., Conover, W.J., 2000. A Comparison of Three Methods for Selecting Values of Input Variables in the Analysis of Output from a Computer Code. Technometrics 42, 55-61. DOI: $10.2307 / 1268522$.

Me, W., Abell, J.M., Hamilton, D.P., 2015. Effects of hydrologic conditions on SWAT model performance and parameter sensitivity for a small, mixed land use catchment in New Zealand. Hydrol. Earth Syst. Sci. 19, 4127-4147. DOI: 10.5194/hess-19-4127-2015

Medeiros, I.C., Silva, R.M., 2014. Análise da erosão hídrica na região semiárida da paraíba usando o modelo SWAT acoplado a um SIG. São Paulo, UNESP, Geociências 33, 3, 457-471.

Moriasi, D.N., Arnold, J.G., Van Liew, M.W., Bingner, R.L., Harmel, R.D., Veith, T.L., 2007. Model evaluation guidelines for systematic quantification of accuracy in watershed simulations. Transactions of the ASABE 50, 885900.

Neitsch, S.L., Arnold, J.G., Kiniry, J.R., Williams, J.R., 2011. Soil and Water Assessment Tool Theoretical Documentation: Version 2009. U.S. Department of Agriculture-Agricultural Research Service, Grassland, Soil and Water Research Laboratory and Texas AgriLife Research, Blackland Research Center, Texas Water Resources Institute Technical Report N. 406. Texas A\&M University System, College Station, TX. 2011. Disponível em: http://swatmodel.tamu.edu/documentation/ Acesso: 08 ago. 2016.

Nossent, J., Bauwens, W., 2012. Multi-variable sensitivity and identifiability analysis for a complex environmental model in view of integrated water quantity and water quality modeling. Water Science \& Technology 65, 539549. DOI: 10.2166/wst.2012.884.

Nossent, J., Elsen, P., Bauwens, W., 2011. Sobol' sensitivity analysis of a complex environmental model. Environmental Modelling \& Software 26, 1515-1525. DOI: $10.1016 / j$.envsoft.2011.08.010

Pereira, D.R., Martinez, M.A., Pruski, F.F., Silva, D.D., 2016. Hydrological simulation in a basin of typical tropical climate and soil using the SWAT model part I: Calibration and validation tests. Journal of Hydrology: Regional Studies 7, 14-37. DOI: 10.1016/j.ejrh.2016.05.002

Pereira, D.R., Almeida, A.Q., Martinez, M.A., Rosa, D.R.Q., 2014a. Q. Impacts of deforestation on water balance components of a watershed on the Brazilian East Coast. Revista Brasileira de Ciência do Solo 38, 1350-1358. DOI: 10.1590/S010006832014000400030

Pereira, D.R., Martinez, M.A., Almeida, A.Q., Pruski, F.F., Silva, D.D., Zonta, J.H., 2014b. Hydrological simulation using SWAT model in headwater basin in Southeast Brazil. Eng. Agríc. 34, 789-799. DOI: 10.1590/S0100-69162014000400018

Pontes, L.M., Viola, M.R., Silva, M.L.N., Bispo, D.F.A., Curi, N., 2016. Hydrological Modeling of Tributaries of Cantareira System, Southeast Brazil, with the Swat Model, Engenharia Agrícola 36, 6, 1037-1049.

Praskievicz, S., Chang, H., 2009. A review of hydrological modelling of basin-scale climate change and urban development impacts. Progress in Physical Geography 33, 650-671. DOI: 10.1177/0309133309348098

Rodrigues, E.L., Elmiro, M.A.T., Braga, F.A., Jacobi, C.M., Rossi, R.D., 2015. Impact of changes in land use in the flow of the Pará River Basin, MG. Rev. bras. eng. agríc. ambient. 19, 70-76. DOI: 10.1590/1807-1929/agriambi.v19n1p70-76

Santos, J.Y.G., Silva, R.M., Carvalho Neto, J.G., Montenegro, S.M.G.L., Santos, C.A.G., Silva, A. M., 2014. Assessment of land-use change on streamflow using GIS, remote sensing and a physically-based model, SWAT. Proceedings of the International Association of Hydrological Sciences (PIAHS) 364, 38-43.

Santos, J.Y.G., Silva, R.M., Carvalho Neto, J.G., Montenegro, S.M.G.L., Santos, C.A.G., Silva, A.M., 2015. Land cover and climate change effects on streamflow and sediment yield: a case study of Tapacurá River basin, Brazil. Proceedings of the International Association of Hydrological Sciences (PIAHS) 371, 189-193. DOI: 10.5194/piahs-371189-2015 
Sartori, A., Lombardi Neto, F., Genovez, A.M., 2005. Classificação Hidrológica de Solos Brasileiros para a Estimativa da Chuva Excedente com o Método do Serviço de Conservação do Solo dos Estados Unidos Parte 1: Classificação. Revista Brasileira de Recursos Hídricos 10, 4, 05-18.

Sarrazin, F., Pianosi, F., Wagener, T., 2016. Global Sensitivity Analysis of environmental models: Convergence and validation. Environmental Modelling \& Software 79, 135-152. DOI: 10.1016/j.envsoft.2016.02.005

Schmalz, B., Fohrer, N., 2009. Comparing model sensitivities of different landscapes using the ecohydrological SWAT model. Adv. Geosci. 21, 91-98. DOI: 10.5194/adgeo-21-91-2009

Schuol, J., Abbaspour, K.C., 2006. Calibration and uncertainty issues of a hydrological model (SWAT) applied to West Africa. Advances in Geosciences, European Geosciences Union 9, 137143, 2006.

Silva, B.M., Silva, E.A., Oliveira, G.C., Ferreira, M.M., Serafim, M.E., 2014. Plant-available soil water capacity: estimation methods and implications. Rev. Bras. Ciênc. Solo 38, 464-475. DOI: 10.1590/S0100-06832014000200011
Tirogo, J., Jost, A., Biaou, A., Valdes-Lao, D., Koussoubé, Y., Ribstein, P., 2016. Climate Variability and Groundwater Response: A Case Study in Burkina Faso (West Africa). Water 8, 171. DOI: 10.3390/w8050171

Van Leiw, M., Veith, T., Bosch, D.D., Arnold, J.G., 2007. Suitability of SWAT for the conservation effects assessment project: comparison on USDA Agricultural research service watersheds. Journal of Hydrologic Engineering ASCE 12, 173-189.

Veith, T.L., Van Liew, M.W., Bosch, D.D., Arnold, J.G., 2010. Parameter sensitivity and uncertainty in SWAT: a comparison across five USDA-ARS watersheds. Transactions of the ASABE 53, 14771486.

Wu, K., Johnston, C.A., 2007. Hydrologic response to climatic variability in a Great Lakes Watershed: A case study with the SWAT model. Journal of Hydrology 337, 187-199. DOI: 10.1016/j.jhydrol.2007.01.030

Xiao, Q., McPherson, E.G., Ustin, S.L., Grismer, M.E., Simpson, J.R., 2000. Winter rainfall interception by two mature open-grow trees in Davis, California. Hydrological Processes 14, 763$784 . \quad$ DOI: $\quad 10.1002 /($ SICI)10991085(200003)14:4<763::AID-HYP971>3.0.CO;27 